\title{
托卡马克边条件对低杂波传播的影响
}

\author{
马中芳 李大丰 陈 激
}

(中国科学院等离子体物理研究所)

\section{一、引 言}

低杂波加热是大型托卡马克辅助加热可能的重要手段之一，许多托卡马克装置都用 Brambilla 关于调相波导组的理论 ${ }^{[1]}$ 来设计波导系统. 但 Brambilla 的波导理论没有考虑托卡 马克隔板附近必然存在的密度快变,我们假定了一个更真实的模型,具体数值计算了隔板处等 离子体密度跳跃对波导塊合的影响. 发现密度快变区存在大的波反射, 严重影响了波导的堣 合效率并在密度快变区和真空壁之间造成驻波场, 这种驻波场可能引起表面加热. 在隔板处 透射的能流有相当多的部分属于快波, 因此能够到达等离子体中心被吸收的慢波功率是非常 有限的. 在高密度运行的装置中, 密度快变将更陡, 因而上述问题将更严重. 此外, 在一定的 条件下，隔板处的反射会使能流谱分布出现多峰，但适当选择参数可对能流谱分布加以控制. 最后,密度的快变还可能放宽可近性条件.

\section{二、物理 模型}

我们在波导、真空和等离子体低密度区采用与 Brambilla 类似的做法 ${ }^{[1]}$, 只要知道等离子 体表面的波阻抗 $Z\left(k_{11}\right)$, 便可求得波导的功率反射系数和沿 $x$ 方向能流的谱分布.

对于 $k_{\| \prime}>k_{0}$ 的波,边界的阻抗值为

$$
Z\left(k_{I I}\right)=\frac{I_{\frac{1}{3}}\left(\zeta_{P}\right)-\rho_{1} I_{-\frac{1}{3}}\left(\zeta_{P}\right)}{I_{-\frac{2}{3}}\left(\zeta_{P}\right)-\rho_{1} I_{-\frac{2}{3}}\left(\zeta_{P}\right)}, \quad \zeta_{P}=\frac{2}{3}\left|k_{\perp}\right| / q^{2} .
$$

Brambilla 假定满足可近性条件的波通过截止点后不再反射, 给出 $\rho_{1}=-e^{-i \pi / 3}$ 。在我们的情 况下, $\rho_{1}$ 应由隔板处的边条件来定, 不再是常数.

我们假设隔板阴影部份是具有线性密度分布的低密度区,隔板处存在密度的跳跃, 隔板内 侧的高密度区具有平缓的密度分布. 为

用通常的低密度近似波动方程 ${ }^{[1]}$ 可求解低密度区中 $E_{z}-B_{y}$ 偏振的慢波, 隔板处 $E_{z} / B_{y}$ 值

$$
\begin{gathered}
Z_{s}=\left.\frac{E_{z}}{B_{y}}\right|_{x_{L}}=i \frac{\left|k_{\perp}\right|}{k_{0}} \frac{J_{\frac{1}{3}}(\zeta)+\rho_{1} J_{-\frac{1}{3}}(\zeta)}{q \zeta^{\frac{1}{2}}\left(J-\frac{2}{3}(\zeta)-\rho_{1} J_{\frac{2}{3}}(\zeta)\right)}, \\
\zeta=\frac{2}{3}\left|k_{\perp}\right| q\left(x_{L}-x_{c}\right)^{\frac{3}{2}} .
\end{gathered}
$$

假定波导口处 $E_{y}-B_{z}$ 偏振的快波为零, 则隔板处的 $E_{y}-B_{z}$ 值为 


$$
Z_{t}=\left.\frac{E_{y}}{B_{z}}\right|_{x_{L}}=-\frac{k_{0}}{k_{x}^{f}} \frac{1-e^{z i k_{x}^{f} x_{L}}}{1+e^{z i k_{x}^{f} x_{L}}},
$$

其中 $k_{x}^{f}$ 是快波沿 $x$ 方向的局部波数.

在高密度区，采用冷等离子体的 WKB 解 ${ }^{[2]}$. 对于满足可近性条件的波，假定波在等离子 体中心被完全吸收,略去反射波: 慢波取反向波而快波取正向波,并相应选取虚部使波在 $x$ 方 向衰减. 隔板处高密度值取得足够大，使高密度区不存在汇合点反射的问题，于是隔板处的 $E_{y} / B_{z}$ 值及 $E_{z} / B_{v}$ 值可分别表为

$$
\begin{gathered}
\left.\frac{E_{y}}{B_{z}}\right|_{x_{I}}=\frac{G_{+} / k_{s}^{\frac{1}{2}} W_{+}-\rho_{2} / k_{f}{ }^{\frac{1}{2}} W_{-}}{-k_{s}^{\frac{1}{2}} G_{+} / k_{0} W_{+}-\rho_{2} k_{f}^{\frac{1}{2}} / k_{0} W_{-}}, \\
\left.\frac{E_{z}}{B_{y}}\right|_{x_{l}}=\frac{1 / k_{s}^{\frac{1}{2}} W_{+}+\rho_{2} G_{-} / k_{f}^{\frac{1}{2}} W_{-}}{\left(-\lambda_{1} k_{s}^{\frac{1}{2}} / \lambda_{2} k_{0} W_{+}-i \lambda_{1} G_{+} / k_{0} k_{s}^{\frac{1}{2}} W_{+}\right)+\rho_{2}\left(\frac{\lambda_{1} G_{-} k_{f}^{\frac{1}{2}}}{\lambda_{2} k_{c} W_{-}}+i \frac{\lambda_{1}}{k_{0} k_{f}^{\frac{1}{2}} W_{-}}\right)} .
\end{gathered}
$$

令电磁波阻抗在隔板处连续便可求得 $Z\left(k_{\prime \prime}\right)$.

\section{三、结果与分析}

数值计算结果显示了以下三个主要特点:

\section{1. 高的功率反讨系数}

图 1 给出不同参数下波导功率反射系数和波导位相差的关系. 波导反射系数有了显著的 增加. 这种增长是由波在隔板密度跳跃处的反射所造成的.

图 2 是图 1 中曲线 $a 、 b 、 c$ 所对应的反射波能流轨迹图,这 三条曲线在隔板的反射大致相同，但因能流偏转不同从而得到 不同的波导反射系数. 可见，小的波导反射系数并不一定表示 波能进到等离子体中心.

由于我们采用了密度跳跃的模型，从而得到的结果十分突 出. 真实托卡马克隔板附近只会存在密度快变的过渡层而不会 有绝对的跳跃, 我们假定快变区密度是指数分布, 用低密度波动 方程近似求解,发现宽度为 $2 \mathrm{~cm}$ 的快变区功率反射仍高达 $40-$ $50 \%$.

\section{2. 能橎的多结构}

图 3 给出能流的谱分布,曲线 $c$ 在慢波段又出现了一个峰, 使功率被转移到慢波段。

这种现象也是隔板反射造成的，反射波在隔板和真空壁之

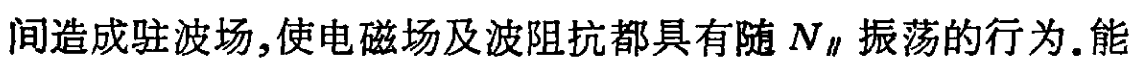
流谱公式可表为

$$
\bar{s}_{x}=\frac{c}{2}\left|E_{z}^{v}\right|^{2} \frac{k_{0}}{k_{\perp}} \frac{l_{m} Z\left(k_{\| 1}\right)}{\left|Z\left(k_{11}\right)\right|^{2}},
$$

其中 $\left|E_{z}^{v}\right|$ 是波导口电场振幅, 它是由波导参数决定的函数, 它 和 $I_{m} Z\left(k_{\| 1}\right) /\left|Z\left(k_{\|}\right)\right|^{2}$ 这两个量都具有随 $N_{\|}$衰减振荡的行

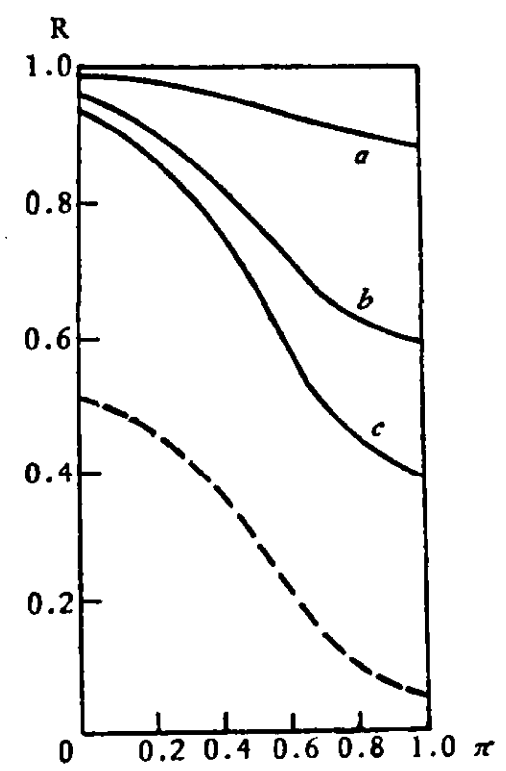

图 1 波导反射系数和相邻 波导位相差的关系 a. 入射频率 $\omega=6.15_{10} 9$ 弧/秒, 低 密度区密度梯度 $\nabla n_{e}=3{ }_{10} 10 \mathrm{~cm}^{-4}$, 隔板到等离子体边界距离 $x_{L}=2 \mathrm{~cm}$, 隔板处的高密度 $n_{L}=1_{10} 13 \mathrm{~cm}^{-3}$; b. $\nabla n_{e}={ }_{10} 11 \mathrm{~cm}^{-4}$. 其它同 $a$; c. $x_{L}=4 \mathrm{~cm}$. 其它同 $a$;

虚线是没有隔板的情况 为, 由于 $k_{0} /\left|k_{1}\right|$ 具有单调下降的性质, 所以能流谱是衰减的, 只有当两个振荡周期相近或相 


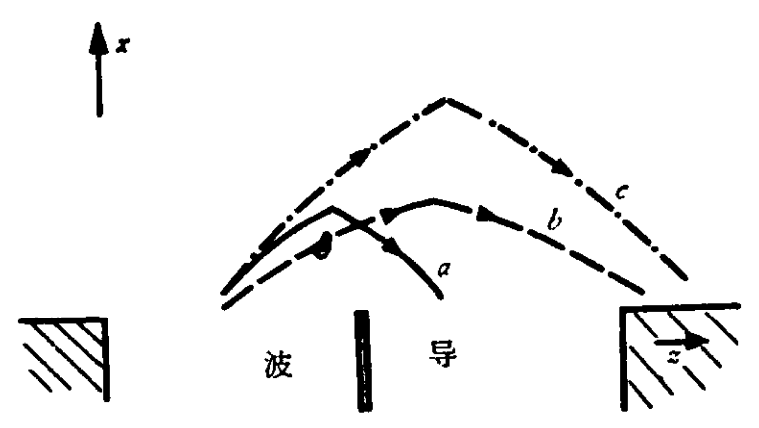

图 2 反射波的射线轨迹图 曲线 $a, b, c$ 对应图 1 的参数

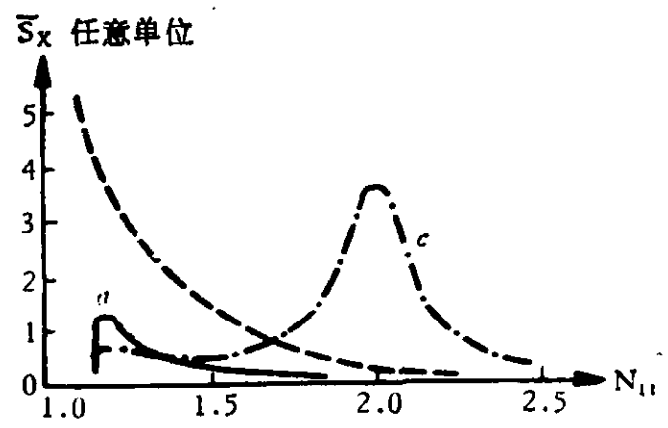

图 3 能流的谱分布曲线 $a 、 c$ 对应图 1 的参数, 虚线是没有隔板的情况

差很大时才能显见第二个峰. 选择波导参数使两周期重合可使曲线 $a$ 在 $N_{\|}=6$ 的慢波段又 出现一个峰.

\section{3. 可近性条件的放宽}

按我们计算的参数, 满足 Stix-Голант 可近性条件 ${ }^{[3]}$ 的临界值是 $N_{c}=1.16$, 但计算结果 表明当隔板高密度值取 $2 \times 10^{13} \mathrm{~cm}^{-3}$ 时,在 $N_{\| /}=1.03$ 时就出现了能流,说明这些本来不可近 的波进到了等离子体中心,这种放宽也是由密度跳跃造成的.

对于一般的平缓密度分布来说，不满足可近性条件的波在消失区的衰减长度比消失区实

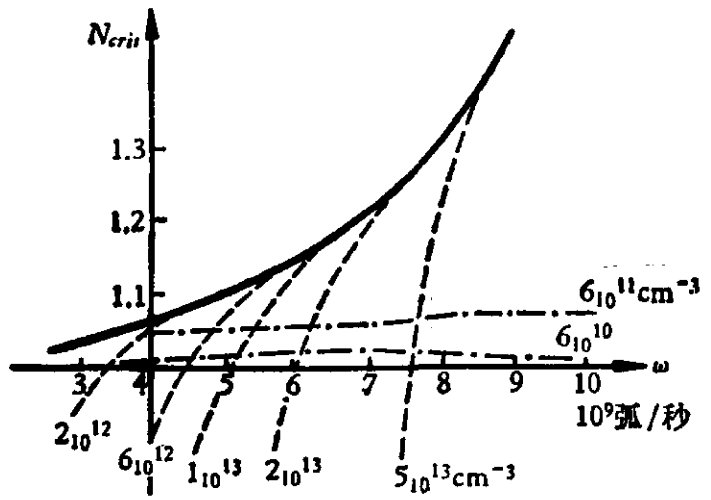

图 4 密度跳跃宽了的可近性条件 际长度短得多, 所以波是不可近的. 但在密度跳跃 模型中，消失区落在跳跃间隔中的波可以从传播的 低密度区直接进人可传播的高密度区,成为可近的, 使可近性条件得到放宽.

根据波在传播过程中不出现消失波的条件可以 得到更普遍的可近性条件，由此可定量讨论密度跳 跃造成的可近性的放宽. 图 4 给出磁场为 3 万高斯 时不同入射频率可近性条件的放宽。实线是无跳跃 时的可近性条件，点划线和虚线分别代表不同高密 度和低密度值的传播条件，放宽了的可近性条件应 是这两个传播条件中较大的值.

致谢：本工作承蒙蛋裕平同志指导,汪茂泉同志参加了有益的讨论,特此致谢.

\section{考文 嗝}

[1] Brambilla, M., Nucl. Fusion, 76(1976), 1:47.

[2] Brambilla，M., 第三届环形装置等离子体加热会议, 1976,84.

[ 3 ] Golant, V. E., Zh. Tekh. Fir., 41 (1971), 2492. 